\title{
Wave Speed and Reflections Proximal to Aneurism and Stenosis of Flexible Tubes *
}

\author{
Wisam S. Hacham, Najdat N. Abdulla, A.Salam Al-Ammri, Ashraf W. Khir
}

\begin{abstract}
Arterial aneurysm and stenosis are disorders that lead to circulation malfunction. Stenosis often leads to hypoxia of the organ depending on the affected artery, whilst aneurism can lead to dissection with known lethal consequences. On both cases, the pulse wave produced by the contracting heart is reflected at these discontinuities, and estimating the size of these reflected waves using wave intensity analysis (WIA) is the main aim of this work. We also aim to measure wave speed, or pulse wave velocity (PWV) as more commonly known within the discontinuities.

We manufactured 4 stenosis and 4 aneurism silicon sections, connected one at a time to a mother tube, and tested in vitro. Pressure and flow were measured proximal to the discontinuity and were used to calculate WIA. PWV was calculated using the foot to foot technique and also the classical Moens-Korteweg and Bramwell-Hill equations. Wave speed in an aneurism decreases, whereas it increases in a stenosis, all compared to the values determined in a standard mother tube. Presence of aneurisms resulted in a backward expansion whilst the presence of stenosis resulted in a backward compression wave, which related linearly to the size of the discontinuity. Larger aneurisms and smaller stenosis cause an increase in wave reflection.
\end{abstract}

\section{INTRODUCTION}

An aneurism is an excessive localized enlargement of an artery caused by a weakening of the arterial lumen wall, while an abnormal narrowing of an arterial segment is called stenosis. Abnormalities due to aneurisms and stenosis present discontinuities that disturb the normal functioning of the systemic circulation and affect the primary goal of the arterial system, which is the perfusion of human body organs. An important consequence of the existence of these discontinuities is the reflection of waves traveling from the heart towards the periphery. These reflected waves are known to increase ventricular mechanical load, and it is therefore desirable to develop techniques for assessing the reflections at such discontinuity.

Wave intensity analysis (WIA) is a technique that is useful for studying cardio-arterial interaction and has the benefit of being a time-domain technique ${ }^{1}$. WIA also allows for the separation of the measured pressure and velocity waves into their forward and backward direction ${ }^{2}$. In essence,

* Research supported by Ministry of Higher Education \& Scientific Research of Iraqi government and Brunel Institute for Bioengineering of Brunel University.

Wisam S. H. is with Al-Khawarizmi College of Engineering, University of Baghdad, Baghdad, Iraq, (e-mail: wisam@kecbu.uobaghdad.edu.iq).

A. N. Najdat is with Mechanical Engineering Department, University of Baghdad, Baghdad, Iraq, (e-mail: najdat@yahoo.co.uk).

M. A. AbdulSalam is with Al-Khawarizmi College of Engineering, University of Baghdad, Baghdad, Iraq, (e-mail: mansour1955@yahoo.com).

A. W. Khir is with Brunel Institute for Bioengineering of Brunel University (phone: +44(0) 1895265857; e-mail: Ashraf.khir@brunel.co.uk). wave intensity (dI) is a hemodynamic expression that represents the energy flux carried by the wave per cross sectional area of the vessel. This amount is equal to the product of the changes in pressure and the changes in velocity across the wave-fronts. The solution of conservation equations of the one dimensional equations of mass and momentum by the method of characteristics for the simultaneous measurements of pressure and velocity is the basis of this time domain analysis. In this technique, the separation of waveforms requires knowledge of the wave speed, which can be determine using one of several techniques such as the traditional foot-to-foot method, PUloop $^{3}$, lnDU-loop ${ }^{4}$. In fact it has been shown that the PU-loop can be used to determine simultaneously both wave speed and the arrival time of reflected wave ${ }^{5}$. Wave speed can also be determined using the classical equations of Bramwell-Hill and Moens-Korteweg equations.

The abnormality in the properties and shape of the arterial lumen will produce reflected wave whose nature depends on the type of discontinuity. The reflection in a discontinuity with converging wall is likely to be positive and the forward compression wave (FCW) would be reflected as a backward compression wave $(\mathrm{BCW})$. Likewise, the reflection in a discontinuity with an increase in size of the arterial lumen is likely to be negative and the FCW will be reflected as a backward expansion wave (BEW).

In this work, we investigate whether the reflection produced by the aneurism will be negative and whether the reflection produced by the stenosis will be positive. Further, whether reflected wave intensity is related to the size of the aneurism and stenosis is the other aim of this work.

\section{METHODS}

\section{A. Wave Intensity Analysis}

The solution of Euler's conservation equations of mass and momentum for the one dimensional homogenous, inviscid and incompressible fluid flow in elastic tubes by the method of characteristics is the basis of WIA. Wave intensity (dI) can be defined as

$$
d I=d P d U
$$

where, $\mathrm{dP}$ and $\mathrm{dU}$ are the changes in pressure $(\mathrm{P})$ and velocity (U) between each two consecutive data points, respectively.

The water hammer equations in the forward (+) and backward (-) directions can be expressed as

$$
d P_{ \pm}= \pm \rho c d U_{ \pm}
$$

where, $c$ is the wave speed and $\rho$ is the fluid density.

If we assume waves interact linearly such that

$d P=d P_{+}+d P_{-}, d U=d U_{+}+d U_{-}$ 
It can be shown that

$d P_{ \pm}=\frac{1}{2}(d P \pm \rho c d U), d U_{ \pm}=\frac{1}{2}\left(d U \pm \frac{d P}{\rho c}\right)$

The pressure and velocity waveforms in the (+) and (-) directions can then be determined by integrating the forward and backward travelling changes in pressure and velocity

$P_{ \pm}=P_{o}+\sum_{t=o}^{T} d P_{ \pm} \quad, U_{ \pm}=U_{o}+\sum_{t=o}^{T} d U_{ \pm}$

where $P_{o}$ and $U_{o}$ are the integration constants, which are taken arbitrarily as the initial pressure and velocity. $\mathrm{T}$ denotes the time for one cycle. Wave intensity can then be separated into the (+) and (-) directions.

$$
d I_{ \pm}=\frac{1}{4 \rho c}(d P \pm \rho c d U)^{2}
$$

\section{B. Experimental Setup}

An illustration of the experimental setup is shown in Fig. 1 and the main elements are described below:

Pump: A piston-pump was used to inject approximately 40cc of water to generate an approximately half-sinusoidal wave by moving the piston from the bottom to the top dead center; a forward compression wave.

Mother tube: The mother tube is made of rubber, has $2 \mathrm{~m}$ in length, $2 \mathrm{~mm}$ in thickness and $1.7 \mathrm{~cm}$ in lumen diameter. .Each discontinuity joined the mother tube at $50 \mathrm{~cm}$ away from the inlet. All tubes were immersed into the water at a depth of approximately $2 \mathrm{~cm}$ in a transparent container.

Measurements: Pressure was measured using transducertipped catheter (Gaeltec, Scotland, UK), whilst flow was measured using ultrasound flowmeter and probes (Transonic, HT323, Netherland). The simultaneous pressure and flow measurements were taken at $50 \mathrm{~cm}$ away from the inlet of the mother tube. Data were acquired at $500 \mathrm{~Hz}$ using analogue digital convertor and Labview software (National Instruments, Texas, USA). Home-written programs in Matlab were used for the analysis of the data, off line.

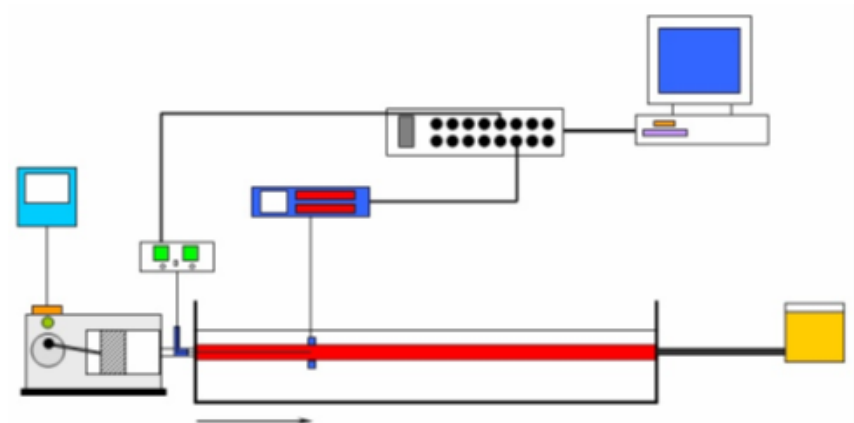

Figure 1. A schematic diagram of the experimental setup including all of the main elements. Mother and daughter tubes connected in line with the pump and a reservoir. All elements of the experiment are on the same horizontal plane, and.the arrow indicates the positive flow direction.

\section{Construction of the Aneurism and Stenosis}

We manufactured eight silicone rubber discontinuities, four to mimic aneurisms and four to mimic stenosis. The maximum inner diameter of the aneurisms was $2.4 \mathrm{~cm}, 3.4 \mathrm{~cm}$, $4.4 \mathrm{~cm}$, and $5 \mathrm{~cm}$, while the minimum inner diameter of the stenosis was $1.3 \mathrm{~cm}, 1 \mathrm{~cm}, 0.5 \mathrm{~cm}$, and $0.25 \mathrm{~cm}$. The design of these discontinuities was made in SolidWorks software and an example of an aneurism and a stenosis is show in Fig. 2.
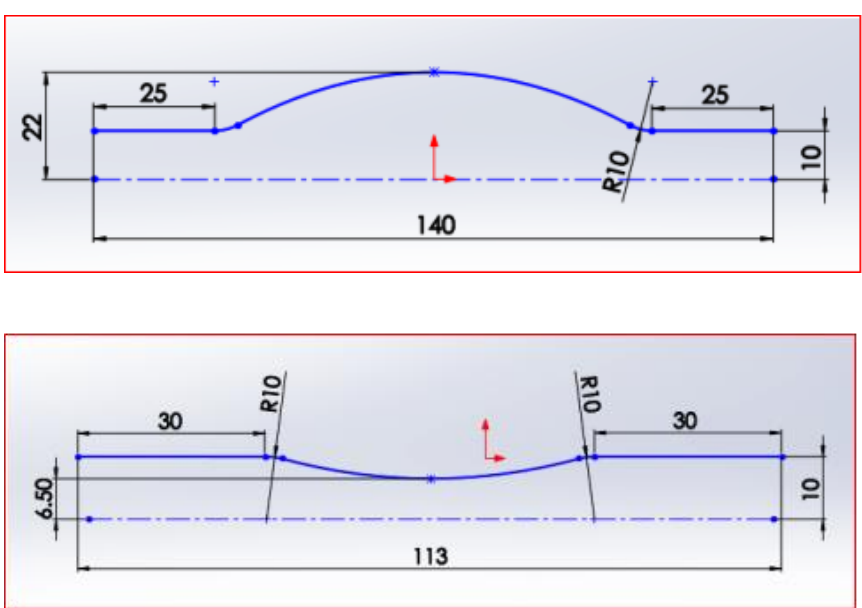

Figure 2. An example of a designed aneurism (top panel) and a stenosis (bottom panel). All dimensions are in milimiters and the drawings show only $1 / 2$ the cross sectional area of each discontinuity.

The manufacturing of the elastic discontinuities was produced by injection molding technique. Liquid silicone was mixed with $2 \%$ catalyst (easycomposites CS2) as a solidifier to produce the artificial silicone rubber parts with $2 \mathrm{~mm}$ in thickness for both the aneurisms and stenosis.

\section{RESULTS}

\section{A. Wave Speeed}

\section{A1. Moens-Korteweg equation}

Estimation of the Young's modulus (E) provides the possibility to employ Moens-Korteweg (M-K) equation to calculate the wave speed (c) through the lumen of the silicone rubber discontinuities. The M-K equation can be written as

$$
c^{2}=\frac{E h}{\rho D}
$$

Where $\mathrm{h}$ is the wall thickness, $\rho$ is density and $\mathrm{D}$ is diameter.

Young's modulus of the mother tube and each of the discontinuities mimicking aneurisms and stenosis were determined using a tensiometer with $500 \mathrm{~N}$ load cell (Instron, UK) with Bluehill2 software. Each specimen was fitted and a tension between $0-70 \mathrm{~N}$ was applied at the rate of $10 \mathrm{~N} / \mathrm{min}$. A relationship between load and strain was established and the slope of which, was used to indicate Young's modulus. Examination of the silicone rubber specimen of $2 \mathrm{~mm}$ thickness, $10 \mathrm{~mm}$ width and diameter for the specimen is 20 $\mathrm{mm}$ resulted in Young's Modulus of 1.3MPa. 
Theoretically, according to the $\mathrm{M}-\mathrm{K}$ equation, the wave will travel with speed equal to $10.4 \mathrm{~m} / \mathrm{s}, 8.7 \mathrm{~m} / \mathrm{s}, 7.7 \mathrm{~m} / \mathrm{s}$ and $7.2 \mathrm{~m} / \mathrm{s}$ in maximum inner diameter aneurisms of $2.4 \mathrm{~cm}$, $3.4 \mathrm{~cm}, 4.4 \mathrm{~cm}$ and $5.0 \mathrm{~cm}$ in, respectively. Similarly, the wave will travel with speed equal to $14.1 \mathrm{~m} / \mathrm{s}, 16.1 \mathrm{~m} / \mathrm{s}, 22.8 \mathrm{~m} / \mathrm{s}$ and $32.2 \mathrm{~m} / \mathrm{s}$ in minimum inner diameter stenosis of $1.3 \mathrm{~cm}, 1 \mathrm{~cm}$, $0.5 \mathrm{~cm}$, and $0.25 \mathrm{~cm}$ respectively.

Young's modulus of the mother tube is equal to $1.3 \mathrm{MPa}$. Using this value and the dimensions of the mother tube in the M-K equation, results in a wave speed of $20 \mathrm{~m} / \mathrm{sec}$.

\section{A2. Bramwell-Hill Equation}

A standard static compliance test was carried out to estimate wave speed in the mother tube, using the BramwellHill equation which can be written as

$$
c^{2}=1 / \rho D_{s}
$$

Where Ds is the distenibility that is defined as the fractional change of the compliance (Cs), with respect to the unstretched cross sectional area $(A), D_{s}=\frac{C_{s}}{A}$. Cs is defined as the fractional change of volume $(\mathrm{dV})$ with respect to the change in pressure $(\mathrm{dP}), \mathrm{C}_{\mathrm{S}}=\frac{\mathrm{dV}}{\mathrm{dP}}$.

The change of pressure in the compression stroke was $101.27 \mathrm{kPa}$ and the volume change corresponding to this difference was 12 milliliters of $\mathrm{H}_{2} \mathrm{O}$ from the initial volume of 45 milliliters of $\mathrm{H}_{2} \mathrm{O}$ as indicated in Fig. 4. Consequently; wave speed is $20 \mathrm{~m} / \mathrm{s}$ when the water is forced through the mother tube of this work.

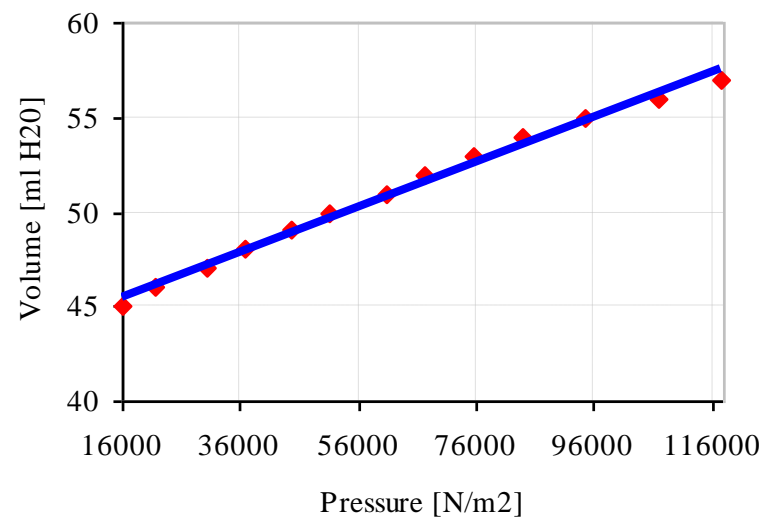

Figure 3. Pressure-Volume diagram for the mother tube.

\section{A3. Foot-to-foot}

To establish wave speed in the stenosis and aneurisms discontinuities we also used the foot-to-foot experimental technique, where two pressure transducers were placed at the inlet and outlet of each discontinuity. With the knowledge of the distance between the two measurement sites and the time that takes the wave to travel from one site to the other, wave speed can be established. The results are shown in Table 1.

\begin{tabular}{|c|c|c|c|c|c|c|c|c|c|}
\hline & $\begin{array}{c}\text { Mother } \\
\text { Tube }\end{array}$ & \multicolumn{3}{|c|}{$\begin{array}{c}\text { Stenosis } \\
\text { Minimum ID }(\mathrm{cm})\end{array}$} & \multicolumn{4}{c|}{$\begin{array}{c}\text { Aneurism } \\
\text { Maximum ID (cm) }\end{array}$} \\
\hline & 1.7 & 0.25 & 0.5 & 1 & 1.3 & 2.4 & 3.4 & 4.4 & 5 \\
\hline $\mathrm{c}(\mathrm{m} / \mathrm{s})$ & 20 & 28 & 22 & 18 & 14 & 13 & 10 & 7 & 6 \\
\hline
\end{tabular}

Table 1. Variation of wave speed within the aneurism and stenosis discontinuities. PWV is higher within the stenosis than the aneurism. As expcetd, the smaller the stenosis the higher the wave speed and the larger the aneurism the lower the wave speed.

\section{B. Wave Reflection}

The reflected pressure waveform was separated from the incident wave using wave intensity analysis (Equation 4, 5). The reflected pressure wave increased with increasing the size of the aneurism and with decreasing the size of the stenosis. The relationship between the reflected wave and the size of the discontinuity is linear with a correlation coefficients of $R^{2}=0.96$ and $R^{2}=0.83$ in the cases of aneurism and stenosis respectively.

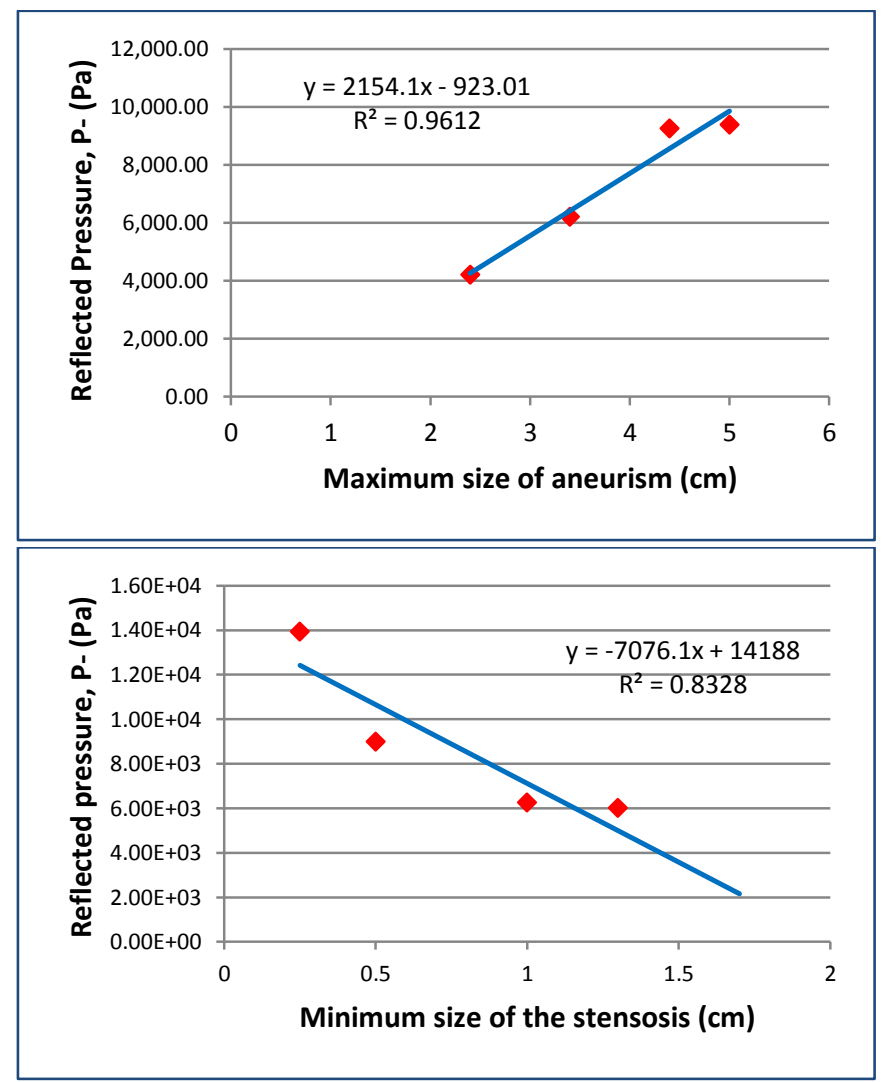

Figure 4. Maximum of the reflected pressure waveform proximal to the aneurisms (top panel) and the stenosis (lower panel) are plotted against the respective sizes of the discontinuities.

Reflected wave intensities (dI-) were calculated using Equation (6) and followed a similar pattern to that of the reflected pressure. An increased size of the aneurism and smaller size of the stenosis resulted in increase in the reflected wave intensity. As shown in Figure 6 the relationship between the reflected intensity and the size of the discontinuity is linear with a correlation coefficients of $\mathrm{R}^{2}=0.95$ and $\mathrm{R}^{2}=0.83$ in the cases of aneurism and stenosis respectively. 


\section{DISCUSSION}

In this in vitro model of the arterial discontinuities of stenosis and aneurism, we have investigated the effect of the size of these discontinuities on the magnitude of reflected waves. We also investigated the wave speed within various models of stenosis and aneurism.

The theoretical estimations of wave speed in the aneurisms and stenosis determined using the $\mathrm{M}-\mathrm{K}$ equation were in a good agreement with those established experimentally using the foot-to-foot technique (table1). Likewise, wave speed calculated using the M-K equation for the mother tube was in a good agreement with that determined using the compliance test and the Bramwell-Hill equation.

The current results have been obtained using invasive measurements of pressure and velocity, which would be difficult to obtain clinically. However, we recently developed a technique for the determination of wave speed and wave intensity using measurements of vessel diameter and flow velocity and tested with human subjects ${ }^{6}$. This is a potentially useful technique since those measurements can be obtained noninvasively using ultrasound imaging modalities in the clinical setting.

\section{CONCLUSION}

Wave speed in an aneurism will decrease, whereas it will increase in a stenosis, all compared to the values determined in a standard mother tube. The pressure wave will be reflected at the aneurism and stenosis, and the size of reflection is closely dependent on the size of the discontinuity. A larger aneurism and a smaller stenosis will generate greater reflections. Further work is required using the noninvasive wave intensity analysis to establish the clinical usefulness and potential of these techniques.

\section{ACKNOWLEDGMENT}

The authors would like to thank Dr Christina Kolyva for her technical assistance. We would also like to thank Drs Xinli Du and Masoud Assadi for their assistance in producing the aneurism and stenosis prototypes.

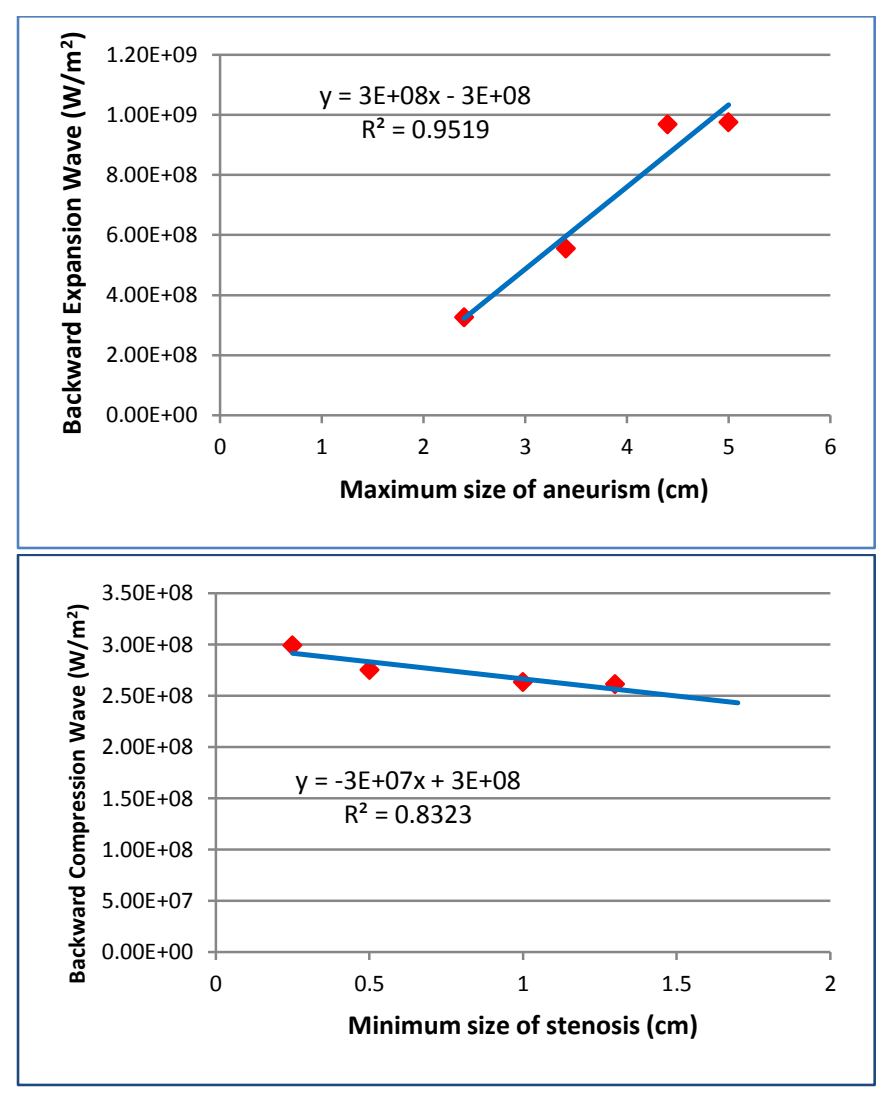

Figure 5. Maximum of the reflected wave intensity proximal to the aneurisms (top panel) and the stenosis (lower panel) are plotted against the respective sizes of the discontinuities.

\section{REFERENCES}

[1] Parker KH. An introduction to wave intensity analysis. Med Biol Eng Compute 47:175-188, 2009.

[2] Parker KH, Jones CJH (1990) Forward and Backward Running Waves in the Arteries: Analysis Using the Method of Characteristics. Journal of Biomedical Engineering 112:322-326.

[3] Khir, AW, O'Brien, A., Gibbs, S, Parker, KH, Determination of wave speed and separation of waves in arteries," Journal of Biomechanics 9, 1145-1155, 2001

[4] Feng J, Khir AW (2010) Determination of wave speed and wave separation in the arteries using diameter and velocity. Journal of Biomechanics 43:455-62.

[5] Khir AW, Swalen MJP and Parker KH. The Simultaneous Determination of Wave speed and the Arrival Time of Reflected Waves in Arteries. Med Bio Eng Comput; 45: 1201-1210, 2007.

[6] Borlotti A, Khir AW, Rietzschel ER et al (2012) Noninvasive determination of local pulse wave velocity and wave intensity: changes with age and gender in the carotid and femoral arteries of healthy human. J Appl Physiol (1985) 113:727-35 